\title{
Das Endoprothesenregister Deutschland am Start
}

Im Jahr 2009 wurden in Deutschland insgesamt knapp 400000 künstliche Gelenke eingebaut, davon 159221 Hüftendoprothesen und 23269 Wechseleingriffe am Hüftgelenk sowie ca. 50000 Hüftendoprothesen bei Schenkelhalsfrakturen. Im gleichen Jahr wurden 148298 künstliche Kniegelenke eingebaut und 11256 Kniegelenke gewechselt. Ein nicht unerheblicher Teil der Wechseloperationen wird jedoch nicht erst 10 bis 15 Jahre nach dem Einbau notwendig, sondern teilweise schon sehr viel früher.

Auf Initiative der Deutschen Gesellschaft für Orthopädie und orthopädische Chirurgie (DGOOC) haben sich daher maßgebliche Beteiligte des Gesundheitswesens, nämlich der AOK Bundesverband, der Verband der Ersatzkassen (vdek), die Prothesenhersteller und das Bundesstelle Qualitätssicherung Institut (BQS) in einem beispielhaften gemeinsamen Projekt über bisher sorgsam getrennte Bereichsgrenzen hinweg zum Aufbau eines deutschen Endoprothesenregisters zusammengeschlossen [1]. Eine derartige Strukturgrenzen-übergreifende freiwillige Partnerschaft ist bisher einmalig. Der gemeinsame Bundesausschuss hat den Aufbau des Endoprothesenregisters ausdrücklich begrüßt.

Die Register gGmbH ist eine $100 \%$ ige Tochter der wissenschaftlichen Fachgesellschaft DGOOC. Durch Nutzung von Routineabrechnungsdaten unter Beachtung der datenschutzrechtlichen Vorgaben wird die notwendige zusätzliche Datenerhebung auf ein Minimum reduziert. Die Implantate werden durch eine weltweit einmalige, von den Herstellern bereitgestellte Produktdatenbank anhand einer Barcode-Erfassung identifiziert. Die Federführung der Fachgesellschaft ermöglicht eine direkte Rückkopplung an die Leistungserbringer und gewährleistet so eine zeitnahe Umsetzung der gewonnenen Erfahrungen zur Verbesserung der Versorgungsqualität [2].

Die Standzeiten von Endoprothesen werden durch die Parameter Implantationszeitpunkt, Prothesentyp sowie Ende der Standzeit durch Explantation oder Tod des Patienten im Rahmen einer prospektiven multizentrischen Registerstudie ermittelt. Die Daten werden weiter aufgeschlüsselt und zeigen, ob die verwendeten Implantate, das operative Vorgehen oder auch patientenspezifische Merkmale mit der erneuten Operation in Zusammenhang stehen. Vor dem Hintergrund der hohen Implantationszahlen in Deutschland und einem hohen Erfassungsgrad der bei AOK und Ersatzkassen versicherten Pati- enten, die ihr Einverständnis gegeben haben, sind repräsentative Aussagen zu erwarten. Das Register wird eine valide Referenzbasis für die Versorgungsqualität in der Endoprothetik darstellen. Der Beginn der Datenerfassung ist für das zweite Quartal 2012 vorgesehen [3].

Autorenerklärung: Der Autor ist ehrenamtlicher Geschäftsführer der gemeinnützigen Endoprothesenregister Deutschland gGmbH. Es bestehen keine finanziellen Interessenkonflikte.

\section{Literatur}

1 Hassenpflug J. Wir brauchen ein Endoprothesenregister. Orthopädie Mitteilungen 2010: 162-163

2 Hassenpflug J. Registerarbeit kann beginnen. Orthopädie Mitteilungen 2011: 139-140

3 Malzahn J, Hassenpflug J. Qualitätskontrolle fürs Kunstgelenk. Gesundheit und Gesellschaft 2011; 14: $21-24$
J. Hassenpflug

Qualitätsmanagement

Schlüsselwörter

Endoprothese

Register

O Implantatdatenbank

Routinedaten

Keywords

endoprosthesis

registry

- implant-database

administrative data

\section{Oadministrative data}

Institut

Orthopädische

Universitätsklinik Kiel

Bibliografie

DOI $10.1055 / \mathrm{s}-0031-1286088$

Dtsch Med Wochenschr 2011; 136: S59 - (c) Georg Thieme Verlag KG Stuttgart · New York . ISSN 0012-0472

Korrespondenz

Prof. Dr. Joachim Hassenpflug Orthopädische

Universitätsklinik Kiel

Michaelisstraße 1

24105 Kiel

Tel. 0432/5972430

Fax 0431/5972456

eMail

hassenpflug@orthop.uni-kiel.de 